\title{
The Comparison of Mandibular Radiomorphometric Indices in Panoramic Radiography between Patients with Chronic Periodontitis and Healthy Individuals
}

\author{
${ }^{1}$ Amir Moeintaghavi, ${ }^{2}$ Hossein Hosseinizarch, ${ }^{3}$ Sara Mohammadzadeh Tabassi
}

\begin{abstract}
Aim: Osteoporosis and periodontitis are two separate diseases with different origins and manifestations. It is believed that these diseases linked together, because they both lead to bone damage, some risk factors are similar, they both have the highest prevalence in middle-aged and older women. Some studies showed that the use of panoramic radiography and special indices could be reliable tools for osteoporosis screening. This study was performed to evaluate the relationship between periodontal disease and jaw osteoporotic indices.
\end{abstract}

Materials and methods: Eighty-two patients with chronic periodontitis and 80 healthy individuals were selected, they had been referred to a private oral and maxillofacial radiology clinic to take a panoramic radiograph. Then panoramic indicators; including the mandibular cortical index $(\mathrm{MCl})$, mental index $(\mathrm{MI})$, and panoramic mandible index (PMI) in both groups were measured, recorded and analyzed.

Results: The mean age of investigated individuals was $39 / 8 \pm$ $9 / 33.58 .6 \%$ of participants were females and $41.4 \%$ were males. $\mathrm{MI}$ and PMI levels in the periodontal group were more than the periodontally healthy group, but the differences between the two groups was not statistically significant $(p=0.808$ and $p=0.102$ respectively). The $\mathrm{MCl}$ level was significantly different between two groups $(p=0.028)$.

Conclusion: The results of this study showed that there is significant relationship between $\mathrm{MCl}$ in panoramic radiography and chronic periodontitis. It is suggested to perform more studies to confirm if this index could be used for screening and indicating of bone status in high risk individuals. This study did not show a strong evidence of a relationship between osteoporosis and periodontitis.

Clinical significance: Since panoramic radiographs are routinely used for screening in dental practice, any association

${ }^{1}$ Professor of Periodontology, ${ }^{2}$ Associate Professor of Oral and Maxillofacial Radiology, ${ }^{3}$ Dentist

${ }^{1}$ Oral and Maxillofacial Diseases Research Center, School of Dentistry Mashhad University of Medical Sciences, Khorasan Razavi, Mashhad, Iran

${ }^{2}$ Dental Research Center, School of Dentistry, Mashhad University of Medical Sciences, Khorasan Razavi, Mashhad, Iran

${ }^{3}$ Private Practice, Mashhad, Iran

Corresponding Author: Sara Mohammadzadeh Tabassi Dentist, Hashemieh Blvd, Sairus Building, Khorasan Razavi Mashhad, Iran, e-mail: sara_mohamadzadet@yahoo.com between radiomorphometric indices of mandible in periodontitis patients might be useful in prediction of osteoporosis in patients referring to dental clinics.

Keywords: Cross-sectional, Periodontitis, Osteoporosis, Radiomorphometric indices.

How to cite this article: Moeintaghavi A, Hosseinizarch $\mathrm{H}$, MohammadzadehTabassi S. The Comparison of Mandibular Radiomorphometric Indices in Panoramic Radiography between Patients with Chronic Periodontitis and Healthy Individuals. J Contemp Dent Pract 2014;15(4):461-465.

Source of support: Mashhad University of Medical Sciences.

Conflict of interest: None declared

\section{INTRODUCTION}

Osteoporosis and periodontitis are two separate diseases with different origins and manifestations. It has been suggested that these diseases could be linked together, because they both lead to bone destruction, they have some similar risk factors; and they both have high prevalence among middleaged and older women. ${ }^{1}$

Panoramic imaging, also referred to as pantomography, is a technique which produces a single tomographic image of 'the facial structures including both the maxillary and mandibular dental arches along with their supporting structures. $^{2}$

Radiographic changes in periodontitis are produced by inflammatory lesions in alveolar bone. Similar to all bone inflammatory lesions, periodontal disease could be associated with both bone resorbtion and bone formation. ${ }^{3}$

Some studies have shown that the use of panoramic radiography and special indices could be reliable tools for the screening of osteoporosis. On the other hand, dental panoramic radiographs are widely used for routine examinations. ${ }^{4-13}$ Some of these indices include: the mental index (MI), the panoramic mandibular index (PMI) and the mandibular cortex index (MCI). ${ }^{14-16}$

Taguchi et al, in order to diagnose postmenopausal osteoporosis, evaluated the usefulness of the width and morphology of the inferior cortex of the mandible on panoramic radiographs. In 29 premenopausal and 95 postmenopausal women, the width and morphology of the mandibular inferior cortex on panoramic radiographs and the 
trabecular bone mineral density (TBMD) of the 3rd lumbar vertebrae (L3) measured using dual energy quantitative computed tomography were compared. Their results suggested panoramic radiography as a reliable technique in screening for osteoporosis. ${ }^{8}$

Klemetti and Kolmakow investigated whether on panoramic radiographs, bone mineral density (BMD) of the mandibular cortex was correlated with a categorization of the morphology of the inferior cortex. In this study, measurements were made on the cortical BMD of the mandible at two sites: (1) buccally and distally from the mental foramen and (2) lingually and distally from the mental foramen. Assessments of the morphology of the mandibular cortex were made on panoramic radiographs, and were categorized into three groups. Based on these findings, the authors recommended the use of an ordinal classification of the mandibular cortex on panoramic radiographs, which might be useful for dentists in assessing the local quality of the cortical bone. ${ }^{7}$

Taguchi et al evaluated the usefulness of measuring the shape of the mandibular inferior cortex and the width on dental panoramic radiographs as a screening tool for spinal osteoporosis in postmenopausal women. The authors recommend that dentists might be able to refer postmenopausal women who are suspected to be suffering from spinal osteoporosis for bone densitometry tests judging by the dental panoramic radiographs. ${ }^{9}$

Jagelaviciene and Kubilius have shown through histomorphometric and microradiographic studies that increases within the porosity of the cortical layer of the mandible led to reductions in bone mass. Orthopantomography is highly useful in estimating the bone density of the mandible, because nearly all patients who attend dental clinics undergo orthopantomography. This provides an opportunity for researchers to investigate osteoporotic changes in the mandible and to identify individuals for further studies, in addition to ensuring good treatment results. ${ }^{17}$

Jagelaviciene et al performed a study with the purpose of investigating the relationship between bone mineral density in the calcaneus (as determined by dual X-ray and laser osteodensitometry techniques) and bone mineral density in the mandible (measured by the panoramic radiomorphometric indices resulting from the application of linear measurements in the panoramic radiographs taken from postmenopausal women). The correlation between the mental index and bone mineral density in the calcaneus in the main group was found statistically significant. Another statistically significant correlation was found between the panoramic mandibular index and bone mineral density in the calcaneus. ${ }^{10}$
Considering the extensive use of panoramic radiography in periodontics and the validity of this method for evaluating the indices of osteoporosis, the aim of this study was to determine if there is any relationship between osteoporosis indices and periodontal disease in panoramic figures.

\section{MATERIALS AND METHODS}

This cross-sectional study spanned an interval ranging from April 2010 to October 2011. The research council and ethical committee of Mashhad University of Medical Sciences approved the protocol for this study, and all the participants signed a written informed consent form prior to their participation.

Inclusion and exclusion criteria in this study included: patients with chronic periodontitis and without any systemic diseases that would affect bone metabolism and possibly cause bone lesions and fractures.

The sample size was calculated equal to 160 patients (80 in each group). All subjects were recruited from patients who had been referred to a private oral and maxillofacial radiology clinic.

All dental panoramic radiographies were prepared with Planmeca X.C (Planmeca, Finland) at 6_8 mA and 18 seconds of exposure time; the kilo-voltage varied between 66 and $70 \mathrm{KV}$. All panoramic radiography images were produced digitally by a computed-radiography software (Konica, Japan; version 110).

Subjects were divided into two groups: the first group (G1) consisted of 82 patients with chronic periodontitis with a mean age of $40.6 \pm 10.00(\mathrm{SD})$ years (20-65 years) and the second group (G2) was made up of 80 periodontally healthy individuals with a mean age of $38.9 \pm 8.55$ (SD) years (2360 years). The participants of both groups were matched in terms of age and gender.

Periodontitis was diagnosed according to the guidelines of the American Academy of Periodontology 1999. ${ }^{18}$

Following this, three dental panoramic radiographic indices, namely the mandibular cortex index (MCI), the mental index (MI) and the panoramic mandibular index (PMI) were calculated on dental panoramic radiographs.

These indices were viewed using a Sony VAIO VGNCS118E (Sony corporation, China) while the monitor was set in an upright position and calculated with the Planmeca Romexis viewer ver. $24.2^{\circledR}$. All measurements were calculated by an expert oral and maxillofacial radiologist.

The student $\mathrm{T}$ test and Mann-Whitney $\mathrm{U}$ tests were used to compare mean values between the two groups. Qualitative data were compared using the Chi-square test. p-values less than 0.05 were considered as statistically significant.

Table 1 shows the definitions of calculated indices. 
The Comparison of Mandibular Radiomorphometric Indices in Panoramic Radiography

Table 1: Radiographic indices and their definition

\begin{tabular}{ll}
\hline Indices & Definition \\
\hline $\mathrm{MCI}^{19}$ & $\begin{array}{l}\text { C1: The endosteal margin of the cortex was even } \\
\text { and sharp on both sides } \\
\end{array}$ \\
& $\begin{array}{l}\text { C2: The endosteal margin showed semilunar } \\
\text { defects (lacunar resorption) and/or seemed to form } \\
\text { endosteal cortical residues on one or both sides } \\
\text { C3: The cortical layer formed heavy endosteal } \\
\\
\text { cortical residues and was clearly porous }\end{array}$ \\
& The thickness of the cortex in mental foramen \\
$\mathrm{H}$ & Distance from inferior border of mental foramen to \\
& the inferior border of mandible \\
$\mathrm{PMI}$ & $\mathrm{MI} / \mathrm{H}$ \\
\hline
\end{tabular}

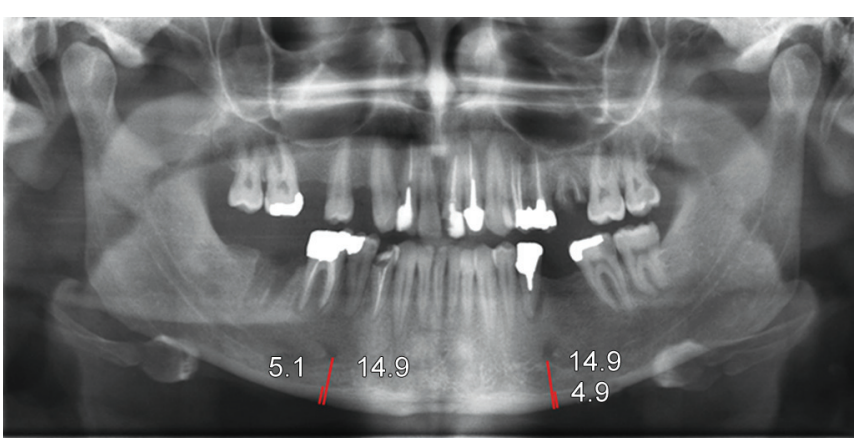

Fig. 1: Panoramic view of a periodontally healthy patient. The short red line represents MI. The high redline shows the $\mathrm{H}$ index (Distance from the inferior border of mental foramen to the inferior border of the mandible)

\section{RESULT}

A total of 162 patients (mean age: $33.9 \pm 8.39$ ) participated in this study. Demographic characteristics has been shown in Table 2. The two groups were matched based on age and gender (Table 2).

\section{MI}

Figure 1 shows the results for the mental index. This index was greater in G1 than in $\mathrm{G} 2$, but there was no statistically significant difference between two groups $(p=0.80)$ (Graph 1 and Fig. 1).

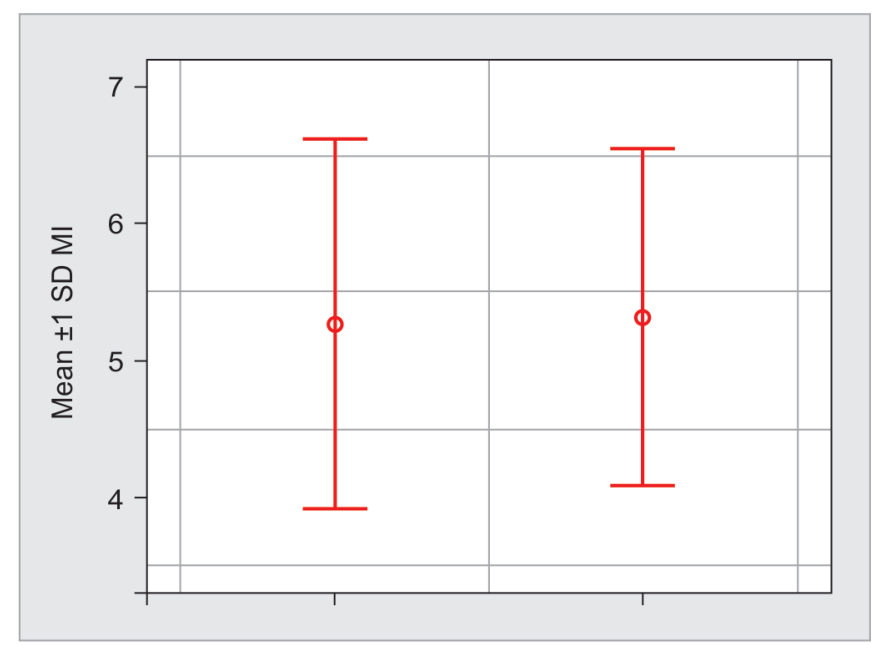

Graph 1: Mental index (Mean $\pm S D$ ) in the two groups
Table 2: Demographic characteristics of the recruited individuals in each group

\begin{tabular}{llll}
\hline & $\begin{array}{l}\text { Healthy } \\
\text { group }\end{array}$ & $\begin{array}{l}\text { Periodontitis } \\
\text { group }\end{array}$ & Total \\
\hline Number & 80 & 82 & 162 \\
Age (Mean \pm SD) & $38.9 \pm 8.55$ & $40.6 \pm 10$ & $39.8 \pm 9.33$ \\
Minimum & 23 & 20 & 20 \\
Maximum & 60 & 65 & 65 \\
PV & $T=1.185$ & $p=0.238$ & \\
Male & $34(42.5 \%)$ & $33(40.2 \%)$ & $67(41.4 \%)$ \\
Female & $46(57.5 \%)$ & $49(59.8 \%)$ & $95(58.6 \%)$ \\
PV & $\mathrm{p}=0.77$ & $\chi^{2}=0.085$ & \\
\hline
\end{tabular}

\section{PMI}

This index was greater in G1 than in G2, but the t-Test did not show any significant difference $(p=0.10)($ Graph 2).

\section{$\mathrm{MCl}$}

C1 was seen mostly in G2, and C2 was seen in the periodontitis group. According to the Chi-square test, there was a significant difference between the two groups $(p=0.02)$. The results of the chi-square analysis can be seen in Table 3 (Figs 2 to 4 ).

\section{DISCUSSION}

Periodontitis is an inflammatory disease of the periodontium with bacterial origins. This condition destroys supportive periodontal tissue and leads to the progressive destruction of periodontal ligaments and alveolar bones. ${ }^{17}$ Osteoporosis is a chronic and progressive reduction of bone mineral density and matrix which leads to bone mass reduction and changes in bone microstructure. ${ }^{20}$ It is believed that these diseases, periodontitis and osteoporosis, are linked together, because they both lead to bone damage; also, some risk factors are similar and both have a high prevalence in middle-aged and older women. ${ }^{17}$ Some studies have shown that changes in sex hormone levels during the periods of adolescence, menstruation, pregnancy

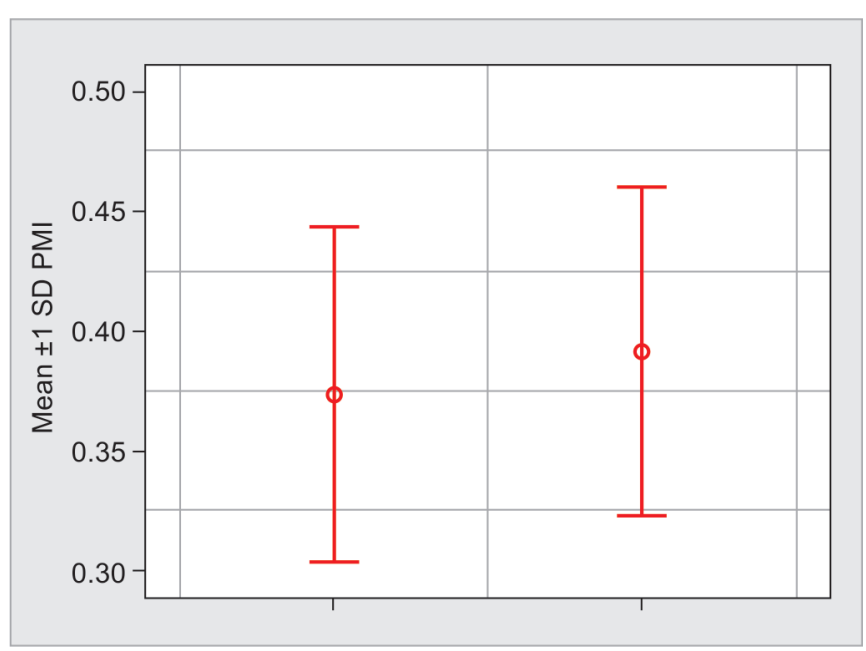

Graph 2: The panoramic mandibular index in the two groups (Mean \pm SD) 
Table 3: Frequency of different types of cortical index in the two groups

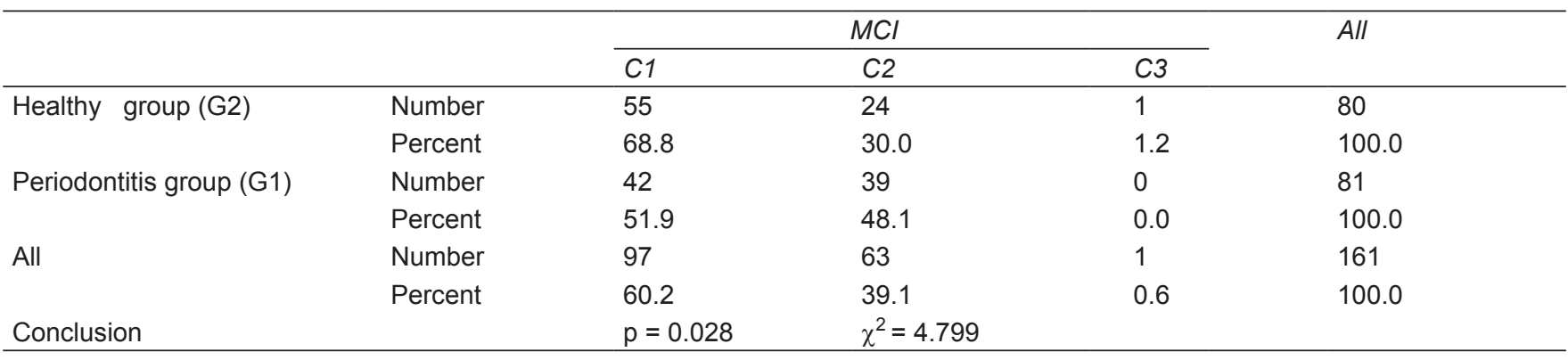

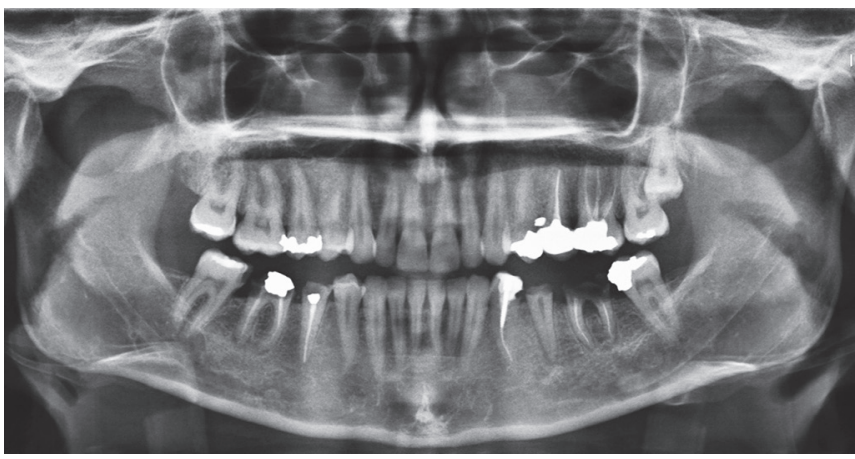

Fig. 2: The panoramic view of a patient with a $\mathrm{C} 1$ type of $\mathrm{MCl}$

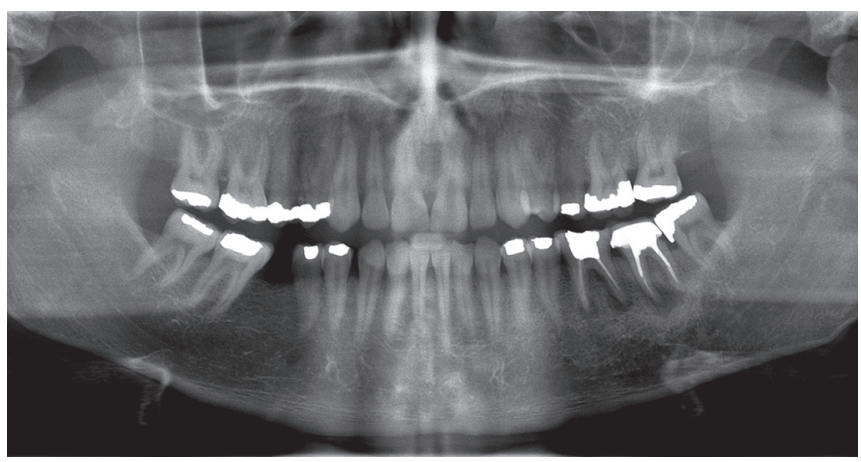

Fig. 4: The panoramic view of a patient with $\mathrm{C} 3$ type of $\mathrm{MCl}$

and menopause cause inflammatory change in gingiva. ${ }^{21,22}$ The beginning of menopause in women is accompanied by a number of changes, such as reduced salivation, the thinning of the oral mucous membrane, gingival recession, increased prevalence of periodontitis and alveolar process resorption. ${ }^{22}$ According to a study by Grodstein, females suffering from osteoporosis who had undergone estrogen therapy were more likely to preserve their teeth, compared to those women who had no history of estrogen therapy. ${ }^{3}$ Payne also showed that postmenopausal women who received substitute hormonal treatment had more healthy periodontium compared to controls. ${ }^{2}$

In spite of studies that have been carried out to show an existing relationship between these two diseases, there are still a few unanswered questions, such as: whether osteopenia in the maxilla or mandible is a local manifestation of osteoporosis with similar etiology and risk factors or it is an independent process that happens due to periodontitis productive factors?

Due to its feasibility and low exposure time, panoramic radiography is a widely used method in dentistry for

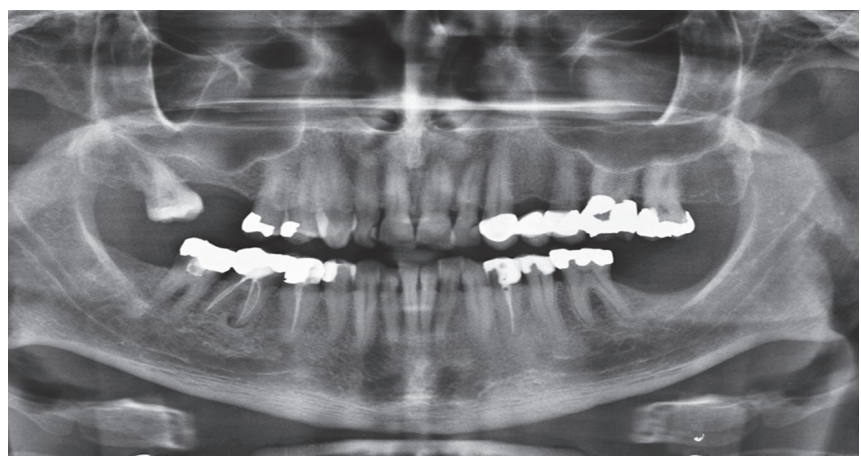

Fig. 3: The panoramic view of a patient with $\mathrm{C} 2$ type of $\mathrm{MCl}$

the screening and overall evaluation of patients with oral diseases. Some studies have shown that the use of panoramic radiography and special indices can be considered as reliable tools for screening osteoporosis in patients referred to a dentist. ${ }^{4-13}$ Some of these indices include: MI, PMI and MCI. Some studies have indicated a significant relationship between bone mineral density and thickness of the mandibular cortex or PMI. ${ }^{14-16}$ Also many studies have shown that MCI is applicable for skeletal bone mineral density. ${ }^{7,8,23,24}$ Mental index has also been recommended for the evaluation of osteoporosis. ${ }^{4}$

In this study, we evaluated these osteoporotic panoramic indices in patients with periodontal diseases (G1) and a group of periodontally-healthy patients (G2).

Our results reveal that there were no significant differences between G1 and G2 in MI and PMI. Mandibular cortex index which is used for the qualitative evaluation of morphologic structures of mandibular cortical layers, was significantly different between the two groups: in G1, the percent of individuals who had the $\mathrm{C} 2$ type of MCI was more than G2 (48.1\% and $31.2 \%$, respectively). We could not draw any conclusions for the $\mathrm{C} 3$ cortical type, since there was just one person with $\mathrm{C} 3$ cortical type in G2. Kalinowski et al showed that the greatest mandibular height was observed in the ages of 30 to 39 in both genders. This height is completely dependent on age and reduces in elders. ${ }^{25}$ Since in our study, there were no differences in mean age between the two groups, periodontitis alone could not have been an influential factor in variations in these indices.

Mandibular cortex index comparison between the two groups showed differences in mandibular cortical 
layer porosity as a result of bone mass reduction. Since periodontitis leads to local connective tissue degeneration and bone destruction, the difference between the two groups could be attributed to periodontitis. Our results, along with those of other studies, showed that MCI might be used as an indicator of mandibular bone loss in osteoporosis or periodontitis, two diseases with connective tissue destruction.

\section{CONCLUSION}

The results of this study showed that there was a significant relationship between the MCI in panoramic radiography and choronic periodontitis. Further studies are required to confirm if this indicator could be used for screening and indicating bone status in high-risk individuals. This study did not show a strong evidence for the existence of a relationship between osteoporosis and periodontitis.

\section{CLINICAL SIGNIFICANCE}

Since panoramic radiographs are routinely used for screening in dental practice, any association between radiomorphometric indices of mandible in periodontitis patients might be useful in prediction of osteoporosis in patients referring to dental clinics.

\section{ACKNOWLEDGMENT}

The results presented in this study have been taken from a student thesis (no. 2573).

\section{REFERENCES}

1. Jagelaviciene E, Kubilius R. The relationship between general osteoporosis of the organism and periodontal diseases. Medicina (Kaunas) 2006;42(8):613-618.

2. Lurie AG. Panoramic imaging. In: White SC, Pharoah MJ. Oral radiology: principle and interpretation. 6th ed. Philadelphia: Elsevier 2009 p. 175.

3. Perschbacher S. Periodontal diseases. In: White SC, Pharoah MJ. Oral radiology: principle and interpretation. 6th ed. Philadelphia: Elsevier 2009 p. 283-289.

4. Cakur B, Sahin A, Dagistan S, Altun O, Caglayan F, Miloglu O, Harorli A. Dental panoramic radiography in the diagnosis of osteoporosis. J Int Med Res 2008 Jul-Aug;36(4):792-799.

5. Vlasiadis KZ, Damilakis J, Velegrakis GA, Skouteris CA, Fragouli I, Goumenou A, Matalliotakis J, Koumantakis EE. Relationship between BMD, dental panoramic radiographic findings and biochemical markers of bone turnover in diagnosis of osteoporosis. Maturitas 2008 Mar 20;59(3):226-233.

6. Tözüm TF, Taguchi A. Role of dental panoramic radiographs in assessment of future dental conditions in patients with osteoporosis and periodontitis. N Y State Dent J 2004 Jan; 70(1):32-35.

7. Klemetti E, Kolmakow S. Morphology of the mandibular cortex on panoramic radiographs as an indicator of bone quality. Dentomaxillofac Radiol 1997 Jan;26(1):22-25.

8. Taguchi A, Suei Y,Ohtsuka M, Otani K, Tanimoto K, Ohtaki M. Usefulness of panoramic radiography in the diagnosis of postmenopausal osteoporosis in women. Width and morphology of inferior cortex of the mandible. Dentomaxillofac Radiol 1996 Nov;25(5):263-267.

9. Taguchi A, Suei Y, Sanada M, Ohtsuka M, Nakamoto T, Sumida H, Ohama K, Tanimoto K. Validation of dental panoramic radiography measures for identifying postmenopausal women with spinal osteoporosis. AJR Am J Roentgenol 2004 Dec; 183(6):1755-1760.

10. Jagelaviciene E, Kubilius R, Krasauskiene A. The relationship between panoramic radiomorphometric indices of the mandible and calcaneus bone mineral density. Medicina (Kaunas) 2010; 46(2):95-103.

11. Horner K, Devlin H. Clinical bone densitometric study of mandibular atrophy using dental panoramic tomography. J Dent 1992;20(1):33-37.

12. Kribbs PJ, Chesnut CH, Ott SM, Kllcoyne RF. Relationships between mandibular and skeletal bone in an osteoporotic population. J Prosthet Dent 1989;62(6):703-707.

13. Mohammad AR, Alder M, McNally MA. A pilot study of panoramic film density at selected sites in the mandible to predict osteoporosis. Int J Prosthodont 1996;9(3):290-294.

14. Benson BW, Prihoda TJ, Glass BJ. Variations in adult cortical bone mass as measured by a panoramic mandibular index. Oral Surg Oral Med Oral Pathol 1991;71(3):349-356.

15. Horner K, Devlin H. The relationships between two indices of mandibular bone quality and bone mineral density measured by dual energy X-ray absorptiometry. Dentomaxillofac Radiol 1998;27(1):17-21.

16. Horner K, Devlin H. The relationship between mandibular bone mineral density and panoramic radiographic measurements. J Dent 1998;26(4):337-343.

17. Sanz M, Newman MG, Quirynen M. Advanced diagnostic techniques. In: Newman MG, Takei HH, Klokkevold PR, Carranza FA. Carranza's clinical periodontology. 10th ed. Philadelphia: Elsevier 2006. p. 579.

18. Flammig TF. Periodontitis. Ann Periodontol 1999 Dec;4(1): 32-38.

19. Klemetti E, Kolmakov S, Kroger H. Pantomography in assessment of the osteoprosis risk group. Scand J Dent Res 1994; 102(1):68-72.

20. Kazanavičius G, Pavilionyte S, Šapokaite G, Kazanavičienė $\mathrm{V}$. The analysis of the changes in mineral bone density in different age groups of females in dual energy roentgenological absorptiometry studies of the spine and ultrasound studies of hucklebone. Endocrinology in Lithuania 2003;11(1):49-53.

21. Tetradis S, Carranza FA, Fazio RC, Takei HH. Radiographic aids in the diagnosis of periodontal disease. In: Newman MG, Takei HH, Klokkevold PR, Carranza FA. Carranza's clinical periodontology. 10th ed. Philadelphia: Elsevier 2006 p. 561.

22. Carranza FA, Takei HH. Clinical diagnosis. In: Newman MG, Takei HH, Klokkevold PR, Carranza FA. Carranza's clinical periodontology. 10th ed. Philadelphia: Elsevier 2006 p. 541-554.

23. Bollen AM, Taguchi A, Hujoel PP, Hollender LG. Case-control study on self-reported osteoporotic fractures and mandibular cortical bone. Oral Surg Oral Med Oral Pathol Oral Radiol Endod 2000;90(4):518-524.

24. Nakamoto T, Taguchi A, Ohtsuka M, Suei Y, Fujita M, Tanimoto K, Tsuda M, Sanada M, Ohama K, Takahashi J, Rohin M. Dental panoramic radiograph as a tool to detect postmenopausal women with low bone mineral density: untrained general dental practitioners' diagnostic performance. Osteoporos Int 2003;14(8):659-664.

25. Kalinowski P, Różyło-Kalinowska I. Panoramic radiomorphometric parameters in Polish patients. Folia Morphol (Warsz) 2011 Aug;70(3):168-174. 